\title{
FGFR2 in gastric cancer: protein overexpression predicts gene amplification and high $\boldsymbol{H}$-index predicts poor survival
}

Soomin $\mathrm{Ahn}^{1,2}$, Jeeyun Lee ${ }^{3}$, Mineui Hong ${ }^{4}$, Seung Tae Kim ${ }^{3}$, Se Hoon Park ${ }^{3}$, Min Gew Choi ${ }^{5}$, Jun-Ho Lee ${ }^{5}$, Tae Sung Sohn ${ }^{5}$, Jae Moon Bae ${ }^{5}$, Sung Kim ${ }^{5}$, Sin-Ho Jung ${ }^{6}$, Won Ki Kang ${ }^{3}$ and Kyoung-Mee Kim ${ }^{1,2}$

${ }^{1}$ Department of Pathology and Translational Genomics, Samsung Medical Center, Sungkyunkwan University School of Medicine, Seoul, Korea; ${ }^{2}$ Center for Companion Diagnostics, The Innovative Cancer Medicine Institute, Samsung Medical Center, Seoul, Korea; ${ }^{3}$ Division of Hematology-Oncology, Department of Medicine, Samsung Medical Center, Sungkyunkwan University School of Medicine, Seoul, Korea; ${ }^{4}$ Department of Pathology, Kangnam Sacred Heart Hospital, Hallym University School of Medicine, Seoul, Korea;

${ }^{5}$ Department of Surgery, Samsung Medical Center, Sungkyunkwan University School of Medicine, Seoul, Korea and ${ }^{6}$ Biostatistics and Clinical Epidemiology Center, Samsung Medical Center, Sungkyunkwan University School of Medicine, Seoul, Korea

\begin{abstract}
FGFR2 gene amplification, and resulting FGFR2 protein overexpression, is rare in gastric cancer patients, and development of an accurate and widely available method for mass screening to identify patients who may respond to treatment with fibroblast growth factor receptor (FGFR) inhibitors is important. We first screened 312 gastric cancer patients with known copy number variations by FGFR2b immunohistochemistry using FPR2-D, an isoformspecific antibody. Next, we performed immunohistochemistry on tissue microarrays from 1574 gastric cancer patients. Selected cases were analyzed for FGFR2 amplification by FISH. In addition, FGFR2b overexpression was studied in $\mathbf{8 8}$ matched primary and metastatic gastric cancers. In the first cohort, FGFR2b immunohistochemistry results correlated very well with those of copy number variation $(r=0.79)$ and FISH $(r=1.0)$. In total, FGFR2b overexpression was identified in 73 of 1974 gastric cancers $(4 \%)$. The concordance between immunohistochemistry and FISH was extremely high; all 2+ and 3+ cases identified by immunohistochemistry were FGFR2 amplified. In the matched primary and metastatic gastric cancer pairs, the positivity and percentage of positive tumor cells were significantly higher in metastatic gastric cancers than in primary gastric cancers $(8 \%$ vs $3 \%$ and $75 \%$ vs $47 \%$, respectively; $\boldsymbol{P}<0.001)$. FGFR2b overexpression was significantly more frequent in gastric cancers with diffuse subtype $(P=0.01)$ and higher $\mathrm{N}$ stage $(P=0.006)$. FGFR2b overexpression with $H$-score $\geq 150$ were independent prognostic factors for overall survival with hazard ratio of 1.836 (95\% confidence interval, $1.034-3.261 ; P=0.038$ ). FGFR2b positivity in immunohistochemistry was strongly correlated with FGFR2 amplification. Given the low frequency of FGFR2 amplification in gastric cancers, FGFRb2 immunohistochemistry is an accurate screening tool to detect FGFR2 amplification, and both primary and metastatic gastric cancer tissues should be tested to select gastric cancer patients for treatment with FGFR2 inhibitors.
\end{abstract}

Modern Pathology (2016) 29, 1095-1103; doi:10.1038/modpathol.2016.96; published online 27 May 2016

Gastric cancer is the second most common cause of cancer-related deaths worldwide, and the prognosis of advanced gastric cancer is still poor. ${ }^{1}$ Several large-scaled clinical trials have failed to demonstrate

Correspondence: Dr J Lee, MD, PhD or Dr K-M Kim, MD, PhD, Department of Medicine, Division of Hematology-Oncology and Department of Pathology and Translational Genomics, Samsung Medical Center, Sungkyunkwan University School of Medicine, 81 Irwon-ro, Gangnam-gu, Seoul 135-710, Korea.

E-mail: jyunlee@skku.edu or kkmkys@skku.edu

Received 14 January 2016; revised 25 April 2016; accepted 25

April 2016; published online 27 May 2016 survival benefits of targeted therapeutic agents in patients with metastatic gastric cancer. Nevertheless, the REGARD trial demonstrated significantly longer progression-free survival of gastric cancer patients treated with ramucirumab, a monoclonal antibody against vascular endothelial growth factor receptor 2, compared with that of patients in the placebo group. ${ }^{2}$ This was the first trial to demonstrate a meaningful benefit for a monotherapy in metastatic gastric cancer. The RAINBOW trial, which compared the efficacy of paclitaxel with or without ramucirumab in second-line chemotherapy, showed prolonged 


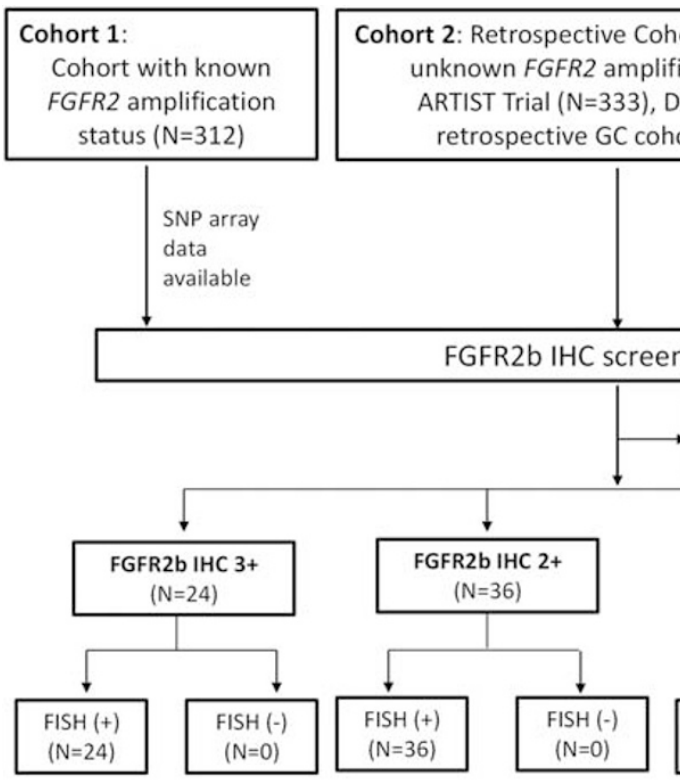

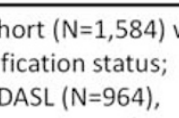

hort $(\mathrm{N}=287)$

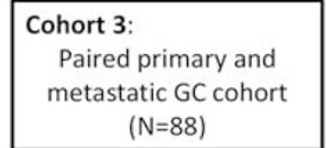

Figure 1 Study design and patient population.

overall survival in the paclitaxel and ramucirumab group. ${ }^{3}$ Thus, after a decade of repeated failures of most targeted agents, except HER2, in showing survival benefits in gastric cancer, these positive trial results have opened a new era for exploring targeted agents for the treatment of gastric cancer. ${ }^{4,5}$

Fibroblast growth factor receptors (FGFRs) are transmembrane tyrosine kinase receptors, and binding of ligands to FGFRs leads to activation of the downstream PI3K-AKT and MAPK-ERK pathways. ${ }^{6}$ Dysregulation of the FGFR2 signaling pathway because of accumulation of epigenetic modifications and genetic alterations is associated with the development and progression of various cancers. ${ }^{7,8}$ Preclinical data suggest that FGFR2 signaling is necessary for tumor lymphangiogenesis and metastatic spread. ${ }^{9}$ Moreover, activation of the FGFR2 pathway is required for the growth of gastric cancers with FGFR2 gene amplification both in vitro and in vivo. ${ }^{10}$ The FGFR2 gene undergoes alternative splicing in the third immunoglobulin domain, leading to two different isoforms of the FGFR2 receptor, FGFR2b and FGFR2c, with different FGF ligand binding. In FGFR2-amplified gastric cancer, it is the FGFR2b isoform that is overexpressed. ${ }^{11}$

In previous studies, the frequency of FGFR2 amplification varied from 3 to $9 \%$ depending on the testing methods. ${ }^{4,5,12-18}$ The prevalence rates also varied between countries- $7 \%$ in the United Kingdom, $5 \%$ in China, and $4 \%$ in Korea-using the same FISH test analyzed in one core laboratory. ${ }^{18}$ Nevertheless, the frequency of FGFR2 overexpression detection by immunohistochemistry ranged from $31 \%$ up to $51 \%$-which were considerably higher than the incidence of FGFR2 amplifica tions. ${ }^{19-22}$
In this large-scale study comprising of +1900 gastric cancer patients, we have used a sensitive and specific monoclonal antibody against the b-isoform of FGFR2, a known driver of gastric cancer. The aims of this study were (1) to assess the correlation between FGFR2b protein overexpression and FGFR2 amplification; (2) to compare FGFR2b protein overexpression between primary and metastatic sites; (3) to evaluate the correlation between $H$-score of FGFR2b expression and prognosis in gastric cancer.

\section{Materials and methods}

\section{Patients and Study Design}

The discovery cohort comprised 312 gastric cancer samples that had been sequenced as previously reported. ${ }^{4}$ In all cases, we performed immunohistochemistry using tissue microarrays. In cases showing FGFR2 copy number gains $(\geq 4)$ in the SNP array analysis and 30 randomly selected FGFR2b immunohistochemistry-negative cases, we performed FISH to validate FGFR2b immunohistochemistry results. After validation, we performed FGFR2b immunohistochemistry in 1584 gastric cancers from four tissue microarray sets from previous studies (Figure 1). ${ }^{23-25}$ Of the 1584 specimens, FGFR2b immunohistochemistry was successfully performed in 1574 cases. The samples were obtained from patients who underwent surgical resection for primary gastric cancer at the Samsung Medical Center, Seoul, Korea, between 1996 and 2006. Finally, we performed immunohistochemistry on the primary gastric cancer- and metastatic lymph node-matched pairs from 88 gastric 

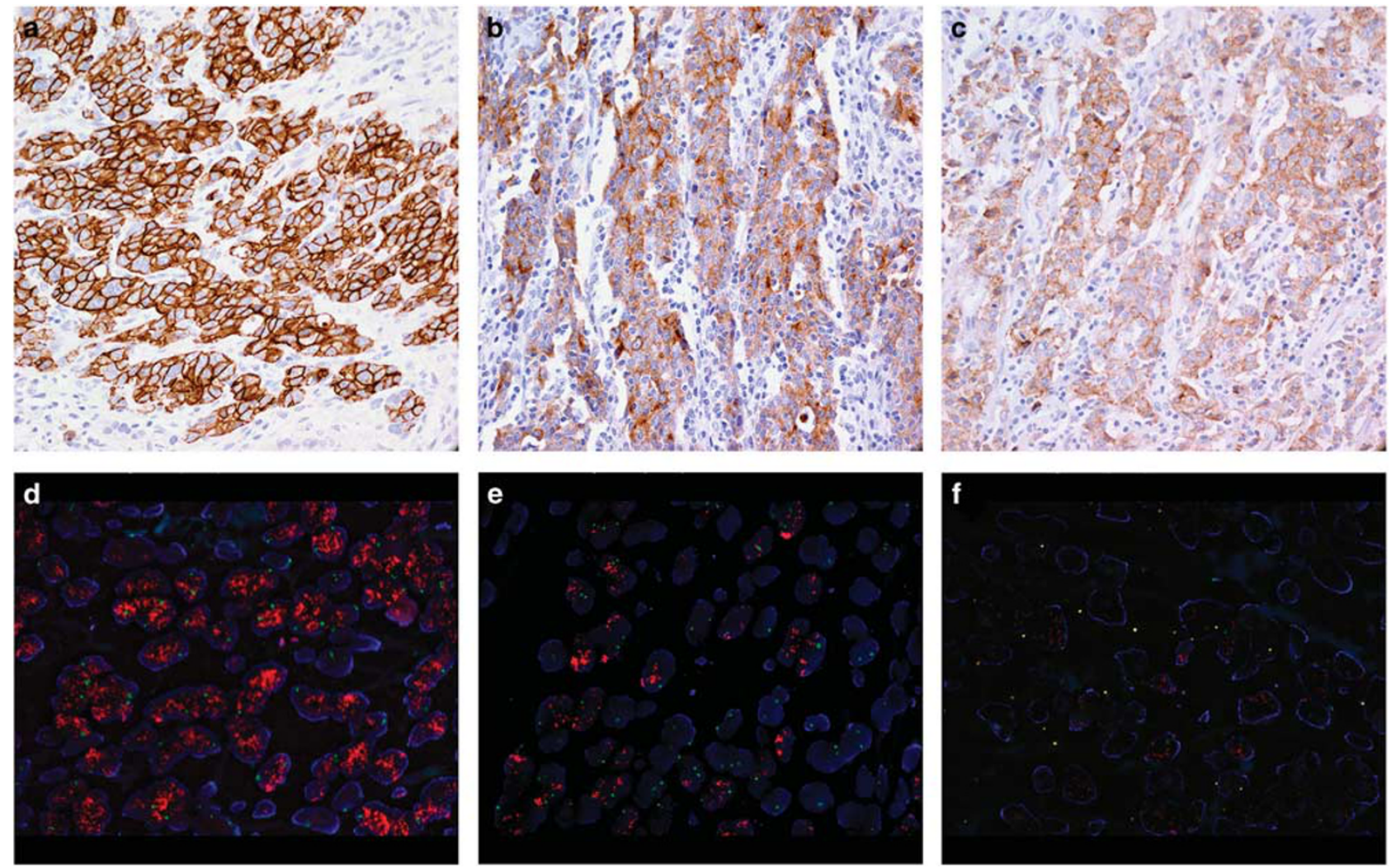

Figure 2 Representative images of FGFR2b expression interpreted by immunohistochemistry as $3+$ (a), 2+ (b), and 1+ (c). Immunohistochemistry results correlated well with copy numbers obtained by FISH. Samples designated as 3+, 2+, and 1+ show high amplification (d), moderate amplification (e), and mild copy number gain (f), respectively.

cancer patients. In cases with FGFR2b overexpression, whole tissue blocks were retrieved for repeated immunohistochemistry and FISH. In addition, we randomly selected 35 immunohistochemistry negative (0) cases from cohort 2 and performed FISH in all cases with entire block. Clinicopathological information, including age, sex, WHO histologic type, Lauren classification, $\mathrm{pT}, \mathrm{pN}$, pM stage, and survival data, was evaluated by reviewing the medical records. Patients lost to follow-up or who died of causes other than gastric cancer were censored for the survival analysis at the last follow-up. The study was approved by the Institutional Review Board of Samsung Medical Center (2014-1-136).

\section{FGFR2b Immunohistochemistry}

FGFR2b immunohistochemistry was performed manually using the primary FGFR2b antibody (FPR2-D provided by Five Prime Therapeutics; 1:200 dilutions). This is a recombinant IgG2a mouse monoclonal antibody that recognizes only the FGFR2b isoform. Formalin-fixed paraffin-embedded

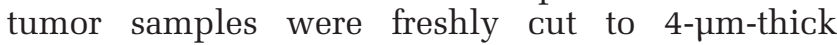
sections and dried at $60^{\circ} \mathrm{C}$ for $30 \mathrm{~min}$. Antigen retrieval was performed for $20 \mathrm{~min}$ using the LOW buffer (pH 6.0) in a $97^{\circ} \mathrm{C}$ water bath. Endogenous peroxidase blocking was conducted for $5 \mathrm{~min}$. Samples were incubated with the primary antibody for $60 \mathrm{~min}$ in room temperature. Subsequently, the sections were incubated with Peroxidase/DAB-10 min K5007 from the DAKO Envision ${ }^{\mathrm{TM}}$ Detection Kit (DAKO, Glostrup, Denmark) for $30 \mathrm{~min}$. The immunohistochemistry results were scored independently by two pathologists (ASM and KMK).

For tissue microarrays with multiple cores, staining in at least a tumor cluster ( $>5$ tumor cells) was regarded as positive. The staining intensity and proportion of positive tumor cells were recorded for whole slides. An intensity score of $3+$ was given when strong membranous and cytoplasmic staining was observed in $\times 4$ magnification (Figure 2a). The score was $2+$ when membranous and cytoplasmic staining was observed in $\times 10$ or $\times 20$ magnification (Figure 2b), and the intensity was rated as $1+$ when weak cytoplasmic staining and/or membranous staining was detected under $\times 40$ magnification (Figure 2c). Immunohistochemistry samples were further analyzed semi-quantitatively using the $H$-score. Briefly, the $H$-score is the sum of the percentage of stained tumor cells multiplied by an ordinal value corresponding to the intensity $(0=$ none, $1=1+, 2=2+$, and $3=3+)$ and ranges from 0 to $300 .{ }^{26}$ 


\section{FGFR2 FISH}

The immunohistochemistry-positive areas of tumor formalin-fixed paraffin-embedded blocks were subjected to FISH. Tumor sections were cut to $1 \mu \mathrm{m}$ thickness, followed by deparaffinization with the pretreatment reagent (Abbott, 30-801250) at $80^{\circ} \mathrm{C}$ for $30 \mathrm{~min}$. Protease digestion procedures were performed using the protease reagent (Abbott, $30-801255)$ at $37^{\circ} \mathrm{C}$ for $20 \mathrm{~min}$. FGFR2 probes (LSI FGFR2 Spectrum Orange Probe, 08N42-020) and CEP 10 (Spectrum Green Probe, 06J37-020) from Vysis (Abbott Molecular, Illinois) were hybridized at $73^{\circ} \mathrm{C}$ for $5 \mathrm{~min}$ and $37^{\circ} \mathrm{C}$ for $20 \mathrm{~h}$. After hybridization, the slides were washed in $2 \times$ saline-sodium citrate/ $0.3 \% \mathrm{NP}-40$ at $72{ }^{\circ} \mathrm{C}$ for $5 \mathrm{~min}$, air dried, and counterstained with 4',6-diamidino-2-phenylindole (DAPI) I and DAPI II (Abbott Molecular). The slides were examined under a fluorescence microscope equipped with Spectrum Texas Red with isothiocyanate and DAPI filters. The FGFR2/CEP 10 ratio were established after counting at least 50 tumor cell nuclei. An FGFR2/CEP 10 ratio higher than 2.0 was interpreted as gene amplification positive. FGFR2 gene copy numbers more than 4 without gene amplification were interpreted as FGFR2 polysomy.

\section{Statistical Analysis}

Statistical analysis was performed using SPSS version 18.0 (SPSS, Chicago, IL, USA) and R software (version 2.12.0 for windows). Correlation between the results of immunohistochemistry, FISH, and Affymetrix SNP 6.0 Arrays was also determined by correlation coefficient test. The average percentage of positive tumor cells in gastric tissues and lymph nodes were compared by Student's $t$-test. Contingency tables and $\chi^{2}$ tests were used to compare the positivity between gastric tissues and lymph nodes in matched pairs. Contingency tables and $\chi^{2}$ tests were also used to correlate FGFR2 amplification status with clinicopathologic variables. Survival was measured from the date of surgery. Overall survival was determined using the Kaplan-Meier method, and survival curves were compared using the log-ratio method. We evaluated the prognostic value of $H$-score every 10 point. Multivariate analysis was performed by Cox proportional hazards regression modeling. A P-value $<0.05$ was considered significant.

\section{Results}

\section{Validation of FGFR2b Immunohistochemistry in the Discovery Set}

In the discovery set $(n=312), 9$ gastric cancers $(3 \%)$ were rated $3+$ for FGFR2b overexpression by immunohistochemistry, and all of these showed FGFR2 gene amplification in FISH. Out of the nine FGFR2b immunohistochemistry-positive gastric cancers, seven showed FGFR2 copy number $\geq 5$ in the SNP array analysis, whereas the remaining two cases showed no copy number gains (Supplementary Table 1). Two FGFR2-amplified and FGFR2b-overexpressed gastric cancer samples were from female patients (aged 52 and 53 years); these samples had a diffuse histologic type by Lauren classification, the tumor cell population was around $60 \%$, and the FGFRb2 immunohistochemistry-positive areas were 30\% and $50 \%$, respectively. Of the 26 cases with FGFR2 copy number $\geq 4$ in SNP array analysis, we found both FGFR2b overexpression and gene amplification in 7 cases with copy number $\geq 5$. However, 19 cases with copy number $<5$ were negative in both immunohistochemistry and FISH (Supplementary Table 2). None of the 30 selected cases with FGFR2b immunohistochemistry score of 0 and no copy number gain in the SNP array analysis showed gene amplification in FISH. The correlation coefficient between immunohistochemistry and FISH was $1.0(P<0.001)$ and between SNP 6.0 array and FISH was $0.79(P<0.001)$.

In addition, immunohistochemistry results for HER2, EGFR, MET, and TP53 were available for analysis in 285 cases of the previously published cohort. ${ }^{4}$ Of 285 patients, HER2 positivity was found in 34 gastric cancers (12\%), EGFR was overexpressed in 14 gastric cancers (5\%), and MET was overexpressed in 9 gastric cancers (3\%). In nine gastric cancers with FGFR2b overexpression, stainings for EGFR, HER2, and MET were all negative, whereas P53 staining was positive in six cases. Epstein-Barr virus was detected in two gastric cancers with FGFR2b overexpression.

\section{FGFR2b Immunohistochemistry and FISH in 1574 Primary Gastric Cancers}

Next, we tested FGFR2 positivity using FGFR2b antibody for immunohistochemistry in 1584 gastric cancer cohort without any information available for FGFR2 amplification (Figure 1). Of the 1584 specimens, FGFR2 immunohistochemistry was successfully performed in 1574 cases. Of these, 4\% $(n=57)$ showed FGFR2b overexpression $(3+, 15 ; 2+, 24 ; 1+$, 18). In all 57 gastric cancers with FGFR2b overexpression, FGFR2b immunohistochemistry and FISH were performed on the entire tumor blocks. In many cases, we observed remarkable intratumoral heterogeneity: out of 57 immunohistochemistrypositive cases, $40(70 \%)$ showed positivity in $<50 \%$ of the tumor volume, and 14 (25\%) showed positivity in $<10 \%$ of the volume. Six of eighteen cases that were scored as $1+$ on tissue microarray analyses were scored as $2+$ or $3+$ in subsequent analysis of the entire tumor sections. Finally, of 1574 cases, 18 cases were rated $3+; 27$ cases, 2+; and 12 cases, 1+.

There was a high correlation between FGFR2 gene amplification by FISH and FGFR2b immunohistochemistry scores (Supplementary Figure 1). 
a

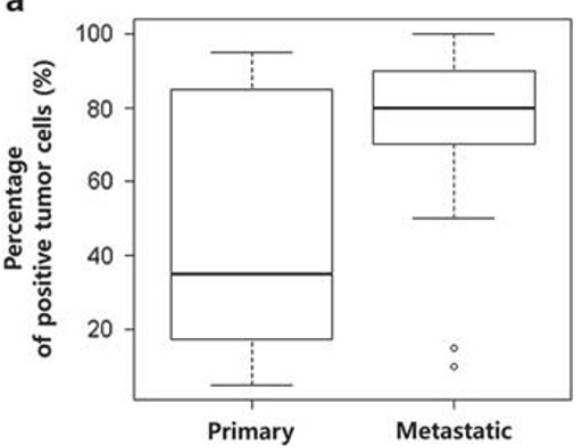

C

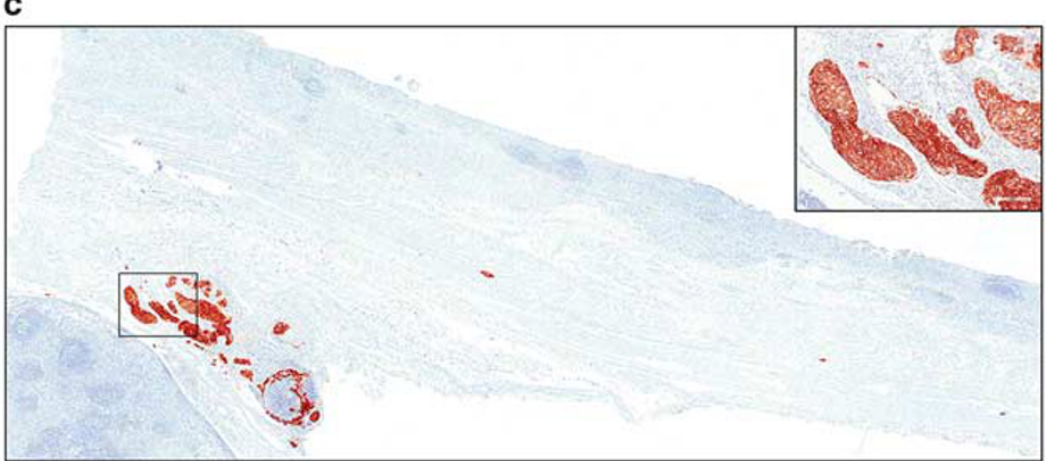

b

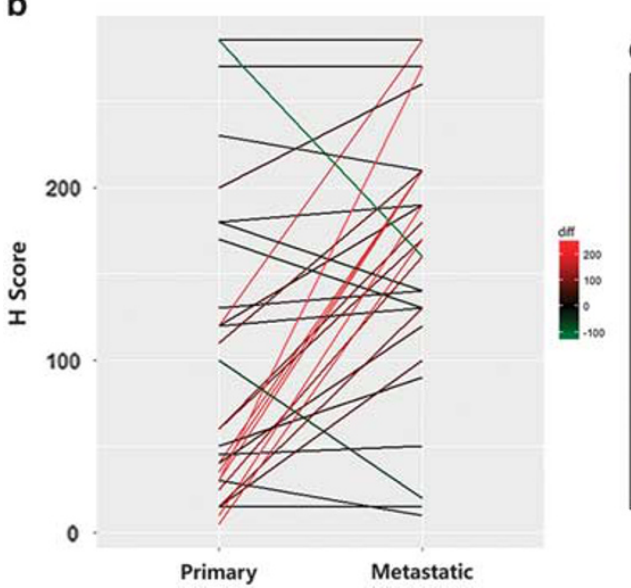

d

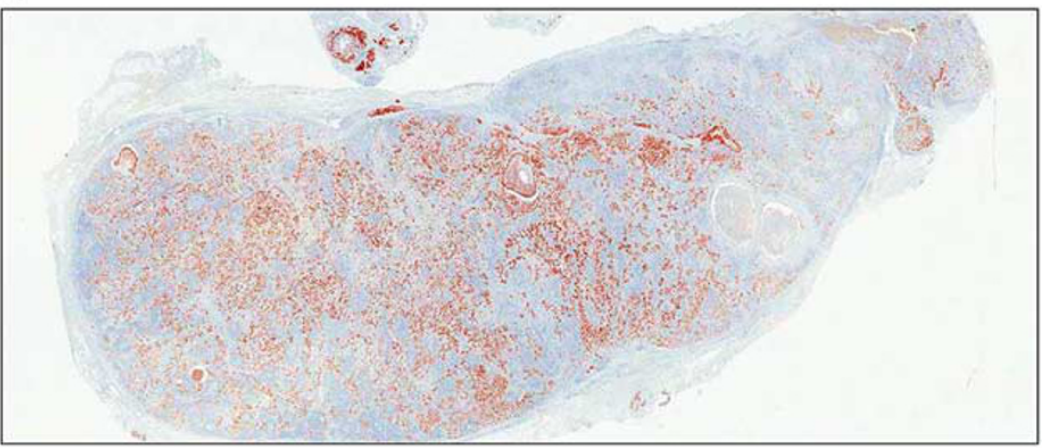

Figure 3 (a) The percentage of immunohistochemistry-positive tumor cells in the lymph node (mean $75 \%$ ) was significantly higher than that in primary gastric cancer tissues (mean $47 \% ; P<0.001$ ). (b) FGFR2b $H$-scores in metastatic lymph node (mean 170) were higher than those in matched primary gastric tissue (mean 103; $P<0.001$ ). Although only lymphatic emboli, a minor population of tumors, showed FGFR2b overexpression in the gastric tissue (inlet indicates higher magnification; c), the majority of metastatic lymph node tumors showed FGFR2b overexpression (d).

Importantly, all gastric cancer patients with FGFR2b $2+$ and $3+$ by immunohistochemistry were confirmed to be FGFR2-amplified by FISH. The average copy numbers ranged from 2 to 100 for FGFR2b immunohistochemistry-positive gastric cancers. The average FGFR2 copy numbers were 64.2 (average FGFR2/CEP 10 ratio: 32.1) for immunohistochemistry $3+$ cases, 38.48 (ratio average: 19.24 ) for 2+ cases, and 10.16 (ratio average: 4.84) for $1+$ cases. Particularly, high FGFR2 amplification (FGFR2/CEP10 $\geq 15$; dark red bar in Supplementary Figure 1) was observed in $94 \%$ of immunohistochemistry $3+$ cases, $48 \%$ of $2+$ cases, and $8 \%$ of $1+$ cases. Six of twelve immunohistochemistry 1+ cases showed no FGFR2 amplification $(n=3)$ or showed polysomy without amplification $(n=3)$. None of the randomly selected 35 cases with FGFR2b immunohistochemistry score of 0 showed FGFR2 amplification by FISH.

In total $(n=1974), F G F R 2$ amplification by FISH was observed in $100 \%$ of the immunohistochemistry $2+$ or $3+60$ cases and $54 \%$ of 13 immunohistochemistry $1+$ cases (Figure 1). Hence, $3 \% \quad(n=67)$ of 1974 gastric cancer patients screened had both FGFR2b protein overexpression ( $1+$ to $3+$ ) and FGFR2 amplification.

\section{FGFR2b Expression in Paired Primary and Metastatic} Gastric Cancers

Eighty-eight matched pairs of primary gastric and metastatic lymph node tissue were evaluated for FGFR2b expression. Seven (8\%) cases showed FGFR2b overexpression in either primary or metastatic gastric cancers; $3(3 \%)$ cases were positive in both primary and the paired metastatic sample; and $4(5 \%)$ cases were positive only in metastatic lymph nodes. Interestingly, FGFR2b overexpression was more frequently observed in metastatic lymph nodes $(8 \%)$ than in primary gastric cancers $(3 \%$; $P<0.001)$.

We extended our study with adding 29 FGFR2bpositive pairs of primary and metastatic tumor from cohort 1 and 2. When evaluating the whole sections of total 32 FGFR2b-positive pairs of primary and metastatic tumor, the percentage of positive tumor cells in metastases (median $80 \%$, mean $75 \%$ ) was significantly higher than that in primary gastric cancer tumor (median 35\%, mean $47 \%$; $P<0.001$; Figure 3a). $H$-scores in metastatic lymph node (median 175, mean 170.2) were also higher than those in matched primary gastric tissue (median 60, mean 103.3; $P<0.001$; Figure 3b). 
Table 1 Clinicopathologic characteristics of 1974 patients with primary gastric cancer according to the FGFR2b overexpression

\begin{tabular}{|c|c|c|c|c|}
\hline \multirow{2}{*}{ Variable } & & \multicolumn{3}{|c|}{ FGFR2b by $I H C$} \\
\hline & & No expression $(\mathrm{n}=1901, \%)$ & Overexpression ( $\mathrm{n}=73, \%)$ & $\mathrm{P}$ value \\
\hline \multirow[t]{3}{*}{ Age (years) } & & & & 0.73 \\
\hline & $<60$ & $1186(62.4)$ & $47(64.4)$ & \\
\hline & $\geq 60$ & $715(37.6)$ & $26(35.6)$ & \\
\hline \multirow[t]{3}{*}{ Sex } & & & & 0.492 \\
\hline & Male & 1246 (65.5) & 45 (61.6) & \\
\hline & Female & 655 (34.5) & $28(38.4)$ & \\
\hline \multirow[t]{7}{*}{ WHO type } & & & & $<0.001^{\mathrm{a}}$ \\
\hline & W/D & $36(1.9)$ & $1(1.4)$ & \\
\hline & $\mathrm{M} / \mathrm{D}$ & $460(24.2)$ & $4(5.5)$ & \\
\hline & $\mathrm{P} / \mathrm{D}$ & $855(45.0)$ & $43(58.9)$ & \\
\hline & Signet ring cell & $418(22.0)$ & $21(28.8)$ & \\
\hline & Mucinous & $71(3.7)$ & $0(0)$ & \\
\hline & Others & $61(3.2)$ & $4(5.5)$ & \\
\hline \multirow[t]{4}{*}{ Lauren classification } & & & & $0.010^{\mathrm{a}}$ \\
\hline & Intestinal & 659 (34.7) & $14(19.2)$ & \\
\hline & Diffuse & $1195(62.9)$ & $56(76.7)$ & \\
\hline & Mixed & $47(2.5)$ & $3(4.1)$ & \\
\hline \multirow[t]{5}{*}{$\mathrm{T}$ stage } & & & & 0.058 \\
\hline & pT1 & $155(8.2)$ & $0(0)$ & \\
\hline & pT2 & $493(25.9)$ & $17(23.3)$ & \\
\hline & pT3 & $1047(55.1)$ & $46(63.0)$ & \\
\hline & pT4 & $206(10.8)$ & $10(13.7)$ & \\
\hline \multirow[t]{5}{*}{$\mathrm{N}$ stage } & & & & 0.006 \\
\hline & pNo & 261 (13.7) & 8 (11) & \\
\hline & pN1 & 599 (31.5) & $16(21.9)$ & \\
\hline & pN2 & 448 (23.6) & $12(16.4)$ & \\
\hline & pN3 & $593(31.2)$ & $37(50.7)$ & \\
\hline \multirow[t]{3}{*}{ M stage } & & & & 0.396 \\
\hline & pMo & 1723 (90.6) & $64(87.7)$ & \\
\hline & pM1 & $178(9.4)$ & $9(12.3)$ & \\
\hline
\end{tabular}

${ }^{\mathrm{a} D o n e}$ by Fisher's exact test.

As we observed significantly increased $H$-scores in metastatic lymph nodes compared with primary gastric cancers, we extended our study using entire tumor blocks from the primary gastric cancers. For this purpose, we selected five FGFR2b-positive gastric cancers with marked $H$-score differences between primary and metastatic tumors ( $H$-score in primary gastric cancer $<50, H$-score in metastatic lymph node $>100$ ). We confirmed the heterogeneity present in different primary tumor blocks with increase of $H$-scores in their metastatic lymph nodes. In detail, $H$-scores were 0-130 (mean 45.6), 0-50 (mean 18.8), 10-160 (mean 61.8), 0-21 (mean 8.5), and 0-30 (mean 10) in primary gastric cancers and 210, 270, 210, 130, and 210 in metastatic lymph nodes, respectively.

Out of 73 cases with FGFR2b overexpression, we found lymphatic tumor emboli in 40 cases. Interestingly, 37 (93\%) of 40 gastric cancer tumor cells within lymphatic tumor emboli showed FGFR2b overexpression. In one case with FGFR2bpositivity in $3 \%$ of tumor volume, the positive staining was localized to the lymphatics (Figure 3c). On the other hand, the majority of metastatic lymph node tumors showed FGFR2b overexpression (Figure 3d).

\section{Clinicopathological Characteristics of FGFR2b- Overexpressed Gastric Cancers}

Clinicopathological characteristics of 1974 patients with primary gastric cancer according to FGFR2b overexpression are described in Table 1. FGFR2b overexpression was significantly more frequent in cases with poorly differentiated $(P<0.001)$ and diffuse type histology as per Lauren classification $(P=0.010)$. The FGFR2 overexpression was more frequent in tumors at higher $\mathrm{pN}$ stages $(P=0.006)$.

There was no significant difference between in overall survival between patients with FGFR2b overexpression and no FGFR2b expression. However, gastric cancer patients with FGFR2b overexpression with $H$-score $\geq 150$ showed significantly shorter overall survival than others $(P=0.001$; Figure 4). In multivariate analyses, high $H$-score $(\geq 150)$ was an independent prognostic factor with hazard ratio of 1.836 (95\% confidence interval, 1.034-3.261; $P=0.038$; Table 2). Clinicopathological features of patients with $H$-score $\geq 150$ are summarized in Supplementary Table 3. Most patients with high FGFR2b expression were relatively young (mean age, 54 years) and their gastric cancer tumors had poorly differentiated WHO histology and a diffuse subtype by Lauren classification. 

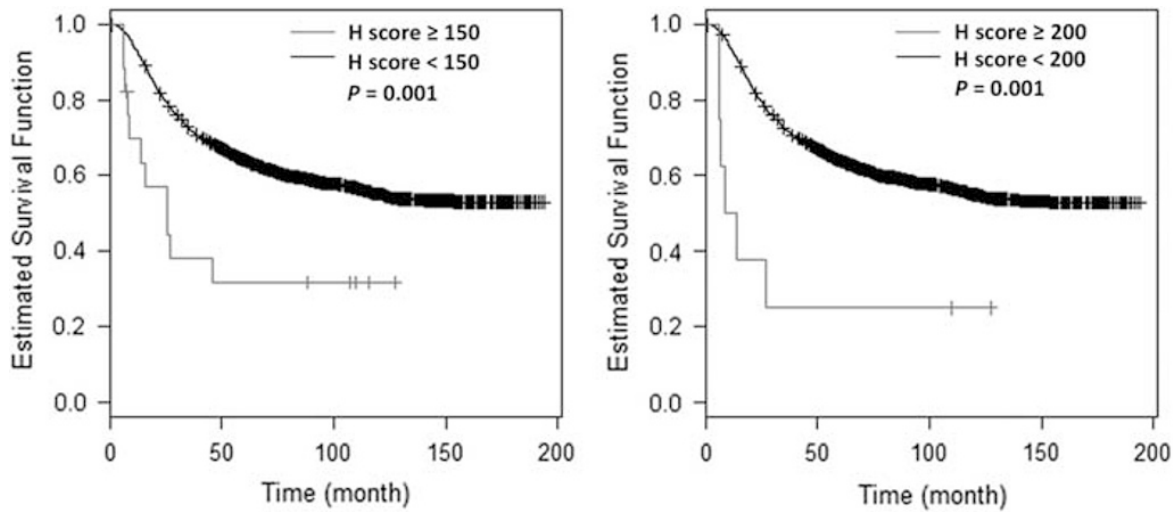

Figure 4 Kaplan-Meier overall survival analysis using FGFR2b $H$-scores. The group with $H$-score $\geq 150$ and $\geq 200$ showed significantly shorter overall survival than groups with $H$-score $<150$ and $<200(P=0.001)$.

Table 2 Multivariate analysis of predictive factors for survival (Cox proportional hazards model)

\begin{tabular}{lll}
\hline Parameters & Odds ratio (95\% CI) & P value \\
\hline T stage & & $<0.001$ \\
T1 vs T2 & $2.089(1.327-3.288)$ & \\
T1 vs T3 & $3.397(2.204-5.234)$ & \\
T1 vs T4 & $5.966(3.790-9.391)$ & \\
N stage & & $<0.001$ \\
$\quad$ N0 vs N1 & $1.307(0.983-1.737)$ & \\
N0 vs N2 & $1.940(1.473-2.556)$ & \\
N0 vs N3 & $3.058(2.363-3.958)$ & \\
FGFR2b IHC $H$-score & & \\
$\quad<150$ vs $\geq 150$ & $1.836(1.034-3.261)$ & \\
\hline
\end{tabular}

Abbreviation: CI, confidence interval.

\section{Discussion}

FGFR2 has emerged as a potential therapeutic target in gastric cancer, and several small-molecule inhibitors and antibodies for FGFRs are currently undergoing clinical trials. ${ }^{27}$ In this study, we used a novel FGFR2b primary antibody to predict gene amplification by immunohistochemistry and found FGFR2b overexpression in $4 \%$ of gastric cancers, and $92 \%$ of these cases were confirmed to be FGFR2 gene amplified by FISH, confirming that it is the FGFR2b isoform, and not the FGFR2c isoform, predominantly expressed in FGFR2-amplified gastric cancer. ${ }^{11}$ We found an excellent correlation between immunohistochemistry and FISH results. Given that several FGFR inhibitors are now in under clinical trials for treatment of metastatic or unresectable gastric cancer harboring FGFR2 amplification or FGFR2b overexpression, our FGFR2b immunohistochemistry can be a powerful tool for massive screening of this small subset of gastric cancer patients.

FISH is the gold standard method to detect gene amplification. However, it is expensive, laborious, and requires specialized fluorescence microscopy equipment. ${ }^{28}$ In contrast, immunohistochemistry is available in most laboratories and offers more advantages in terms of cost-effectiveness. There have been few reports on the correlation between FGFR2 amplification and protein overexpression by immunohistochemistry. ${ }^{16,17}$ However, the previously reported FGFR2 immunohistochemistry results did not perfectly correlate with FGFR2 amplification because almost all of the commercially available FGFR2 antibodies are made to intracellular epitopes and cross react with other FGFRs-like FGFR1. Given the rarity of FGFR2 amplification in gastric cancer, an accurate assay that can be used clinically for massive screening is urgently needed. In the initial screening cohort with SNP array data available, ${ }^{4}$ we found two cases with FGFR2 amplification by FISH and FGFR2b protein overexpression, but no FGFR2 copy number gain in SNP array. Of note, these two FGFR2-amplified and FGFR2boverexpressing gastric cancers had diffuse-type histology with heterogeneous FGFR2b overexpression. This discrepancy between SNP array and FISH may be due to a small population of FGFR2amplified cells within tumors and subsequent contamination with normal cells. Based on our present and previous findings, ${ }^{29}$ FGFR2 screening with immunohistochemistry may be more sensitive to select FGFR2-amplified gastric cancer patients when compared with genomic sequencing, especially in diffuse-type gastric cancer patients. In 19 cases with copy number gain by SNP but negative in both immunohistochemistry and FISH, the estimated copy number in SNP array was $<5$ in all cases. So, we presume that copy number 4 in SNP results needs caution for interpretation.

The prognostic significance of FGFR2 amplification and its association with other clinicopathological factors in gastric cancer patients are varied. ${ }^{13,18}$ In this large-scale study with 1974 gastric cancer patients, FGFR2 amplification and FGFR2b overexpression were more commonly identified in poorly differentiated tumors with diffuse histology, which is different from other reports. ${ }^{4,15}$ Diffuse histology was found in most tumors with high 
$H$-score $\geq 150$. Moreover, we found more frequent FGFR2b expression in higher $\mathrm{T}$ and $\mathrm{N}$ stages, consistent with previous findings examining FGFR2 amplification. ${ }^{15}$ In this study, although FGFR2 amplification itself was not associated with overall survival, FGFR2b overexpression with high $H$-score showed strong prognostic value for survival. Considering the controversial clinical significance and high intratumoral heterogeneity of FGFR2 amplification in gastric cancer, ${ }^{13,18}$ the extent of FGFR2b overexpression, rather than the presence of FGFR2 amplification itself, may be a better predictor of patient survival. Because FGFR2 FISH assays traditionally only examine a small portion of the tumor, whereas immunohistochemistry assays provide data on the entire tumor section, immunohistochemistry may be a better assay to cover intratumoral heterogeneity. Our study demonstrates that patients with high FGFR2b immunohistochemistry $H$-scores had a poorer prognosis than other patients, and these patients would be good candidates for FGFR-targeted therapy.

Based on these results, we propose using FGFR2b immunohistochemistry, instead of FISH, for screening to identify gastric cancer patients harboring FGFR2 amplification for two reasons. First, immunohistochemistry and FISH showed excellent correlation. All $2+$ and $3+$ immunohistochemistry cases showed FGFR2 amplification and staining intensity also correlated with the degree of gene amplification. Second, given the marked intratumoral heterogeneity of FGFR2 amplification, it is important to select positive areas with immunohistochemistry and confirm amplification in these areas by FISH. Performing FISH on randomly selected tumor areas may increase the probability for false-negative results.

Intriguingly, analysis of paired primary and metastatic gastric cancer tumors revealed more FGFR2bpositive tumor cells in metastases than in primary gastric cancer. Considering the marked heterogeneity and low frequency of FGFR2 amplification, this frequency of FGFR2b overexpression within lymphatic tumor emboli is noteworthy. Based on these results and preclinical studies, FGFR2 amplification may play important roles in tumor progression, particularly in lymphangitic metastasis of a subset of gastric cancers, and the potential therapeutic effect from targeted therapy can be expected in patients with this gastric cancer subset. ${ }^{9}$ Moreover, FGFR2b overexpression rate was higher in metastatic lymph nodes than in primary tissue. Despite the small number of cases, $H$-scores in metastatic lymph node were also much higher than those in matched primary gastric tissue. Considering that high $H$-score $(\geq 150)$ indicated poor prognosis and such tumors are expected to respond better to FGFR2 inhibitors, performing immunohistochemistry on investigating lymph nodes might increase the chance of identifying patients with high $H$-score.

This study used tissue microarrays for the first screening of FGFR2 expression. As we observed marked intratumoral heterogeneity present in gastric cancers, the prevalence of FGFR2b overexpression in our study might have been underestimated.

In conclusion, our new FGFR2b immunohistochemistry assay is an accurate screening tool to predict FGFR2 amplification, and whenever feasible, both primary and metastatic gastric cancer tissues should be tested when selecting gastric cancer patients for treatment with FGFR2 inhibitors. Currently, FPA144, an antibody against FGFR2b, is being investigated under a phase I clinical trial with gastric cancer patients who were found positive for FGFR2b overexpression by immunohistochemistry (clinicaltrials.gov; NCT\#02318329).

\section{Acknowledgments}

We thank Kristen Pierce, Robert Sikorski and Julie Hambleton in the Five Prime Therapeutics (South San Francisco, CA, USA) for providing FGFR2b monoclonal antibody. This study was supported by a grant from the 20 by 20 project of Samsung Medical Center (GF01140111).

\section{Disclosure/conflict of interest}

The authors declare no conflict of interest.

\section{References}

1 Jemal A, Bray F, Center MM et al. Global cancer statistics. CA Cancer J Clin 2011;61:69-90.

2 Fuchs CS, Tomasek J, Yong CJ et al. Ramucirumab monotherapy for previously treated advanced gastric or gastro-oesophageal junction adenocarcinoma (REGARD): an international, randomised, multicentre, placebo-controlled, phase 3 trial. Lancet 2014;383:31-39.

3 Hansjochen Wilke W, Eric Van Cutsem, Sang Cheul Oh et al. RAINBOW: A global, phase III, randomized, double-blind study of ramucirumab plus paclitaxel versus placebo plus paclitaxel in the treatment of metastatic gastroesophageal junction (GEJ) and gastric adenocarcinoma following disease progression on first-line platinum- and fluoropyrimidine-containing combination therapy rainbow IMCL CP12-0922 (I4T-IE-JVBE)J Clin Oncol 2014;32 (suppl 3; abstr LBA7):2014.

4 Cristescu R, Lee J, Nebozhyn M et al. Molecular analysis of gastric cancer identifies subtypes associated with distinct clinical outcomes. Nat Med 2015;21: 449-456.

5 Cancer Genome Atlas Research N. Comprehensive molecular characterization of gastric adenocarcinoma. Nature 2014;513:202-209.

6 Katoh M, Katoh M. FGF signaling network in the gastrointestinal tract (review). Int J Oncol 2006;29: 163-168.

7 Davies H, Hunter C, Smith R et al. Somatic mutations of the protein kinase gene family in human lung cancer. Cancer Res 2005;65:7591-7595. 
8 Grose R, Dickson C. Fibroblast growth factor signaling in tumorigenesis. Cytokine Growth Factor Rev 2005;16: 179-186.

9 Larrieu-Lahargue F, Welm AL, Bouchecareilh M et al. Blocking Fibroblast Growth Factor receptor signaling inhibits tumor growth, lymphangiogenesis, and metastasis. PLoS One 2012; 7:e39540.

10 Xie L, Su X, Zhang L et al. FGFR2 gene amplification in gastric cancer predicts sensitivity to the selective FGFR inhibitor AZD4547. Clin Cancer Res 2013;19: 2572-2583.

11 Gemo AT, Deshpande AM, Palencia S et al. FPA144: A therapeutic antibody for treating patients with gastric cancers bearing FGFR2 gene amplification. AACR Annual Meeting 2014;2014, Abstract 5446.

12 Deng N, Goh LK, Wang $\mathrm{H}$ et al. A comprehensive survey of genomic alterations in gastric cancer reveals systematic patterns of molecular exclusivity and co-occurrence among distinct therapeutic targets. Gut 2012;61:673-684.

13 Matsumoto K, Arao T, Hamaguchi T et al. FGFR2 gene amplification and clinicopathological features in gastric cancer. Br J Cancer 2012;106:727-732.

14 Das K, Gunasegaran B, Tan IB et al. Mutually exclusive FGFR2, HER2, and KRAS gene amplifications in gastric cancer revealed by multicolour FISH. Cancer Lett 2014;353:167-175.

15 Jung EJ, Jung EJ, Min SY et al. Fibroblast growth factor receptor 2 gene amplification status and its clinicopathologic significance in gastric carcinoma. Hum Pathol 2012;43:1559-1566.

16 Ooi A, Oyama T, Nakamura $\mathrm{R}$ et al. Semi-comprehensive analysis of gene amplification in gastric cancers using multiplex ligation-dependent probe amplification and fluorescence in situ hybridization. Mod Pathol 2015;28:861-871.

17 Park YS, Na YS, Ryu MH et al. FGFR2 assessment in gastric cancer using quantitative real-time polymerase chain reaction, fluorescent in situ hybridization, and immunohistochemistry. Am J Clin Pathol 2015;143: 865-872.

$18 \mathrm{Su}$ X, Zhan P, Gavine PR et al. FGFR2 amplification has prognostic significance in gastric cancer: results from a large international multicentre study. $\mathrm{Br} \mathrm{J}$ Cancer 2014;110:967-975.
19 Nagatsuma AK, Aizawa M, Kuwata T et al. Expression profiles of HER2, EGFR, MET and FGFR2 in a large cohort of patients with gastric adenocarcinoma. Gastric Cancer 2015;18:227-238.

20 Toyokawa T, Yashiro M, Hirakawa K. Co-expression of keratinocyte growth factor and K-sam is an independent prognostic factor in gastric carcinoma. Oncol Rep 2009;21:875-880.

21 Murase H, Inokuchi M, Takagi Y et al. Prognostic significance of the co-overexpression of fibroblast growth factor receptors 1,2 and 4 in gastric cancer. Mol Clin Oncol 2014;2:509-517.

22 Hattori Y, Itoh $\mathrm{H}$, Uchino S et al. Immunohistochemical detection of K-sam protein in stomach cancer. Clin Cancer Res 1996;2:1373-1381.

23 Lee J, Lim do H, Kim S et al. Phase III trial comparing capecitabine plus cisplatin versus capecitabine plus cisplatin with concurrent capecitabine radiotherapy in completely resected gastric cancer with D2 lymph node dissection: the ARTIST trial. J Clin Oncol 2012;30: 268-273.

24 Yoon RG, Baek JH, Lee JH et al. Diagnosis of thyroid follicular neoplasm: fine-needle aspiration versus coreneedle biopsy. Thyroid 2014;24:1612-1617.

25 Ha SY, Lee J, Kang SY et al. MET overexpression assessed by new interpretation method predicts gene amplification and poor survival in advanced gastric carcinomas. Mod Pathol 2013;26:1632-1641.

26 Detre S, Saclani Jotti G, Dowsett M. A "quickscore" method for immunohistochemical semiquantitation: validation for oestrogen receptor in breast carcinomas. J Clin Pathol 1995;48:876-878.

27 Wang K, Yuen ST, Xu J et al. Whole-genome sequencing and comprehensive molecular profiling identify new driver mutations in gastric cancer. Nat Genet 2014;46:573-582.

28 Selinger CI, Rogers TM, Russell PA et al. Testing for ALK rearrangement in lung adenocarcinoma: a multicenter comparison of immunohistochemistry and fluorescent in situ hybridization. Mod Pathol 2013;26:1545-1553.

$29 \mathrm{Kim}$ ST, Lee J, Hong $\mathrm{M}$ et al. The NEXT-1 (Next generation pErsonalized tX with mulTi-omics and preclinical model) trial: prospective molecular screening trial of metastatic solid cancer patients, a feasibility analysis. Oncotarget 2015;6:33358-33368.

Supplementary Information accompanies the paper on Modern Pathology website (http://www.nature.com/ modpathol) 